\title{
Poolmodell zur Einrichtung von Qualitätsmanagement- systemen - Chance und Herausforderung kleiner und mittlerer Unternehmen
}

\author{
Prof. Dr.-Ing. habil. Gerd Stegemann
}

\section{Problemstellung}

Das Anspruchsniveau an materielle Produkte und Dienstleistungen (Angebotsprodukte) ist national und international durch gestiegene Qualitätsforderungen gekennzeichnet. Die Qualität der Angebotsprodukte ist zu einem der wichtigsten Wettbewerbselemente geworden, wobei unter den Bedingungen gesättigter Märkte kaum mehr eine Branche oder ein Wirtschaftszweig davon unberuihrt bleibt. Langfristiger Erfolg am Markt setzt Qualitätsfähigkeit, hohe Flexibilität und schnelle Reaktionsfähigkeit bei der Realisierung der sich permanent entwickelnden Kundenerwartungen voraus. Im Verdrängungswettbewerb ist damit zunehmend der "Schnelle“ dem „Langsamen“ iiberlegen. Das trifft in gleichem Maße für die herstellende Industrie wie für das Dienstleistungswesen zu. In der ISO 9000-Familie existieren gesonderte Leitfäden für die Gestaltung von QMSystemen der herstellenden Industrie [DIN EN ISO 90041) und des Dienstleistungswesens [DIN ISO 9004-2]. Trotzdem ist aus der Sicht der Gestaltung von Qualitätsmanagementsystemen (QM-Systemen) eine klare Trennung zwischen herstellender Industrie und Dienstleistungswesen häufig nicht möglich, da vielfach eine direkte Verknuipfung von Elementen beider Bereiche erfolgt.

Wie Abb. 1 verdeutlicht, ist die Wirtschaftlichkeit eines Unternehmens wesentlich von seiner Qualitätsfähigkeit abhängig. Grundanliegen aller Tätigkeiten muß es sein, Fehler zu vermeiden statt Fehler zu suchen und zu beseitigen.

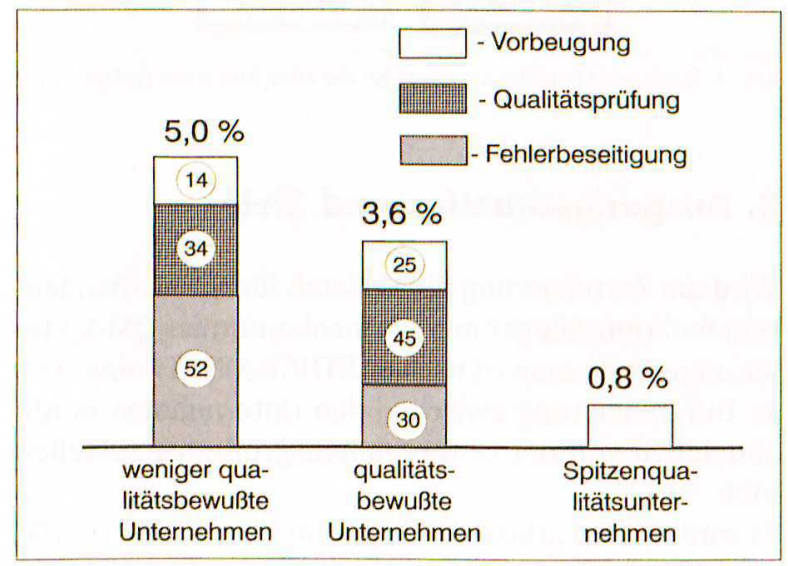

Abb. 1: Aufwendungen von Unternehmen für das Qualitätsmanagement ihrer Produkte (in Prozent des Umsatzes) (2)

Kennzeichen zeitgemäßen Qualitätsmanagements ist deshalb eine alle Bereiche und Tätigkeiten des Unternehmens einschließende Führungstätigkeit zur Qualität, die durch eine permanente Produkt-, Prozeß- und Mitarbeiterorientierung charakterisiert ist und die Erfuillung der Kundenforderungen in den Mittelpunkt aller Bemuihungen stellt. Dadurch wird das Qualitätsmanagement zu einer wichtigen Grundlage langfristigen Geschäftserfolgs zum Nutzen aller Mitarbeiter des Unternehmens sowie der Gesellschaft. Es ist integraler Bestandteil einer modernen Unternehmensphilosophie (Abb. 2).

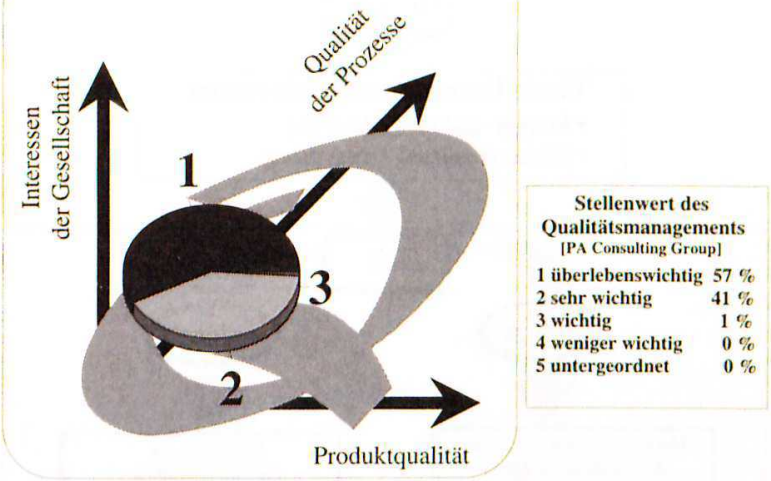

Abb. 2: Dimensionen und Bedeutung der Qualität als Wettbewerbselement

Der Qualitätsbegriff bezieht sich aus dem Grund unter heutigen Bedingungen nicht nur auf die Qualität der Angebotsprodukte (materieller und immaterieller Art) wie z. B. auf deren Fehlerfreiheit oder Zuverlässigkeit. Vielmehr muß der Qualitätsbegriff genauso die Qualität der Herstellungs-/Dienstleistungsprozesse, die Qualität aller vor- und nachgelagerten Prozesse und die Interessen der Gesellschaft einschließen. Qualitätsmanagement geht damit weit uiber die Sicherung der Fehlerfreiheit der Produkte hinaus (Abb. 2).

Letzteres macht für KMU die Verbindung von Qualitätsund Umweltmanagement zwingend erforderlich.

Diesem Anliegen entsprechen die Systematisierung und konsequente Realisierung qualitäts- und umweltrelevanter Tätigkeiten auf der Basis eines integrierten Managementsystems.

Mit der Einrichtung von QM-Systemen werden gewöhnlich interne und externe Ziele verfolgt (Abb. 3).

Von grundlegender Bedeutung für Effizienz und Effektivität des Prozesses der Entwicklung und Realisierung betriebsspezifischer Lösungen des Qualitätsmanagements und deren Strukturierung in normenkonformen QM-Systemen ist die Einstellung, Überzeugung und Motivation der Geschäftsführung zu dieser Aufgabenstellung. Betrachtet die Geschäftsfuihrung Qualitätsmanagement als Führungsaufgabe, dann wird sie mit 
aller Konsequenz die Schaffung dauerhafter Lösungen anstreben und durch das eigene Vorleben die Mitarbeiter in diesem Prozeß befluigeln und mitreißen. In diesem Fall richtet sich die Entwicklung des QM-Systems zunächst im Schwerpunkt auf die Optimierung der Prozesse (leitungsmotivierter Ansatz).

Betrachtet die Geschäftsfuihrung demgegenuiber Qualitätsmanagement mehr oder weniger stark als „Muß“, $z$. B. weil Kunden und/oder Gesetzgeber ein zertifiziertes QM-System fordern (interessenpartnermotivierter Ansatz), dann fehlt häufig die ausreichende Motivation zur Realisierung des anspruchsvollen Prozesses der Einrichtung und Erhaltung eines wirksamen QM-Systems, man fühlt sich „diszipliniert“, in ein abstraktes System gepreßt (z. B. nach der ISO 9000-Familie). Die Lösungen bleiben halbherzig und werden bestimmt durch das „Mindestanspruchsniveau“ des Zertifizierers. Im Vordergrund steht nicht die Gewährleistung der eigenen Qualitätsfähigkeit, sondern die „Befriedigung“ des Kunden/ Gesetzgebers durch das Zertifikat.

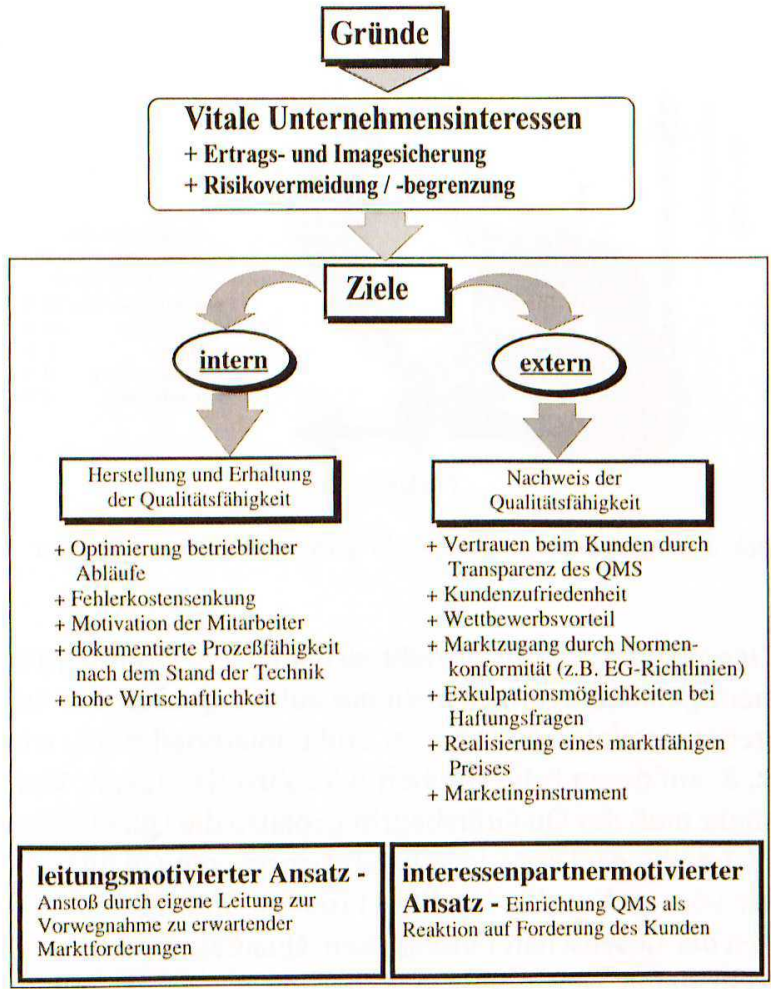

Abb. 3: Gründe und Ziele der Einrichtung von Qualitätsmanagementsystemen

Der Nachweis der eigenen Qualitätsfähigkeit durch die Zertifizierung des QM-Systems ist als wichtiges Marketinginstrument anzusehen, vielfach für Unternehmen auch aufgrund von Forderungen aus Vorschriften (z. B. für Produkte, für die harmonisierte EU-Richtlinien gelten), des Markenschutzes oder Kundenforderungen (trifft insbesondere auch KMU) für den Marktzugang unabdingbar.

Die Einrichtung und Implementierung eines QM-Systems ist eine anspruchsvolle Aufgabe, die abhängig von den betrieblichen Bedingungen im Normalfall zwischen 12 und 24 Monate in Anspruch nimmt. Es zeigt sich, daß dieser Prozeß vielen Unternehmen erhebliche Anstrengungen abfordert und Schwierigkeiten bereitet, oft sogar mit Riickschlägen verbunden ist.
Als wesentliche Ursachenkomplexe dafuir werden weitgehend für KMU unternehmensexterne Spezifika / Hemmnisse (s. Tab. 1) , aber auch unternehmensinterne Bedingungen angesehen (Abb. 4). Selbstverständlich beeinflussen solche "Reibungsverluste" nicht nur den Zeitbedarf für die Einrichtung und Implementierung eines zertifizierungsfähigen QM-Systems in KMU, sondern in entscheidendem Maße auch die personellen und finanziellen Aufwendungen für diesen Prozeß. Die Gestaltung und Implementierung von QM-Systemen ist damit eine Führungsaufgabe.

+ Umfangreiches Geflecht von Gesetzen , Vorschriften und Normen mit daraus entstehenden Problemen beziiglich der Überschaubarkeit der Rechtsbeziehungen

+ Regelwerke zum QM sind sehr allgemein formuliert, unzureichende Berücksichtigung spezieller Gegebenheiten der KMU, es entstehen Probleme für die praktische Übertragung

+ in KMU keine Forschungskapazitäten für die Anpassung / Weiterentwicklung von Methoden / Techniken des QM

+ weitgehend für die Bedingungen der KMU fehlender Methodenund Technikenapparat

+ eng begrenzte personelle Kapazitäten (qualitativ und quantitativ) für die Einrichtung von QMS

+ sehr begrenzte Nutzung der EDV für das Qualitätsmanagement

+ eingeschränkte / fehlende Akzeptanz zur Systematisierung des QM - teilweise Überschätzung der "traditionellen Qualitätsarbeit"

Tab. 1: Spezifika/Hemmnisse bei der Einrichtung und Realisierung normenkonformer QM-Systeme in Klein- und Mittelunternehmen (KMU)

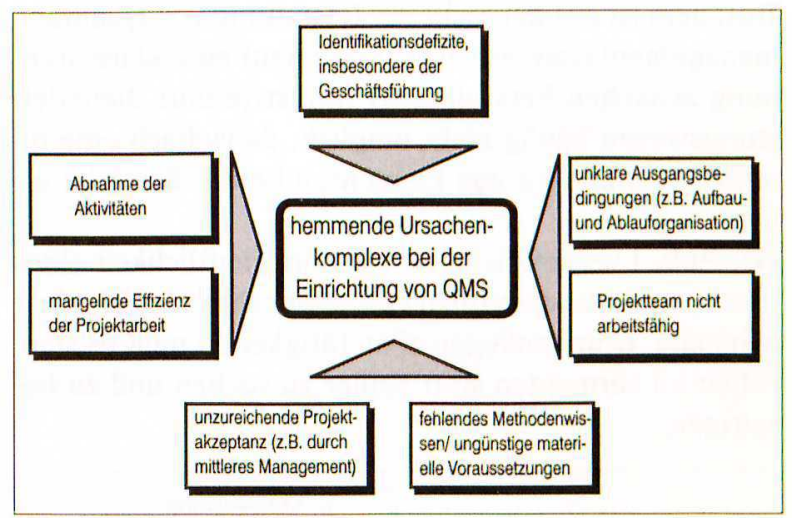

Abb. 4: Hemmende Ursachenkomplexe bei der Einrichtung von QM-Systemen

\section{Ausgangssituation und Ziele}

Wird die Zertifizierung als Maßstab für das Vorhandensein funktionsfähiger und normenkonformer QM-Systeme angesetzt, dann ist nach BAETHGE-KINSKY eine starke Differenzierung zwischen den Unternehmen in Abhängigkeit von der Unternehmensgröße festzustellen (Abb. 5).

Es wird offensichtlich, daß insbesondere in KMU $(<100$ Mitarbeiter) erhebliche Rückstände bestehen.

Eine Analyse des Anteils brandenburger Unternehmen $<100$ Mitarbeiter je Unternehmen fuir das verarbeitende Gewerbe und das Baugewerbe (s. Abb. 6) zeigt, daß mehr als $90 \%$ aller Unternehmen in diese Größenklasse fallen. 


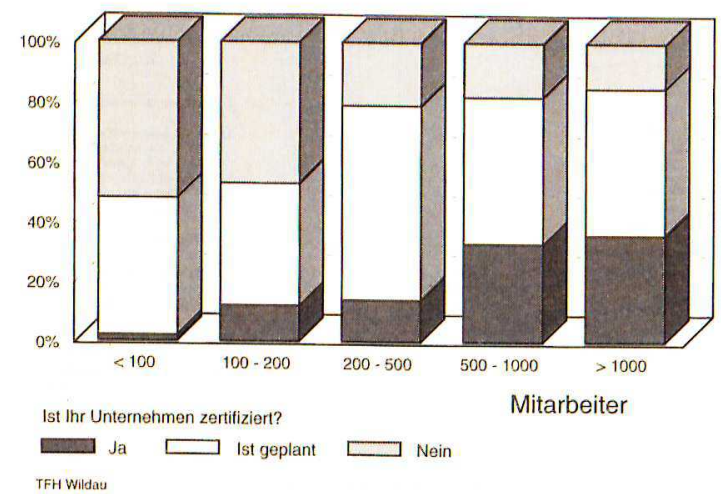

Abb. 5: Stand der Zertifizierung nach DIN ISO 9001 ... 9003 nach Betriebsgrößen. Baethge-Kinsky, V. u.a.: QZ 39(1994)2, S. 97

Forschungsarbeiten des Verfassers bestätigen große Wissensdefizite bezuiglich der Einrichtung und Implementierung normenkonformer QM-Systeme in KMU unter Beachtung der dringend erforderlichen Berücksichtigung der Branchenspezifik in diesem Prozeß.

Untersuchungen des Verfassers zeigen außerdem, daß die effektive Einrichtung und Implementierung von QM-Systemen in KMU (fast) immer einer prozeßnahen Begleitung durch externe Einrichtungen bedarf.

Als eine Möglichkeit zur Lösung dieses Erfordernisses wird die Projektarbeit im Unternnehmensverbund (Poolmodell) angesehen.

Im Sinne dieses Beitrages wird sie als zeitlich befristetes Zusammengehen von KMU einer/verschiedener Branchen in einem Pool, zum Zwecke der Einrichtung von normenkonformen QM-Systemen und deren Zertifizierung im Zusammenwirken mit einer externen Einrichtung und nach dem Prinzip periodischer Beratungen im Pool sowie der individuellen Betreuung der beteiligten Unternehmen angesehen.
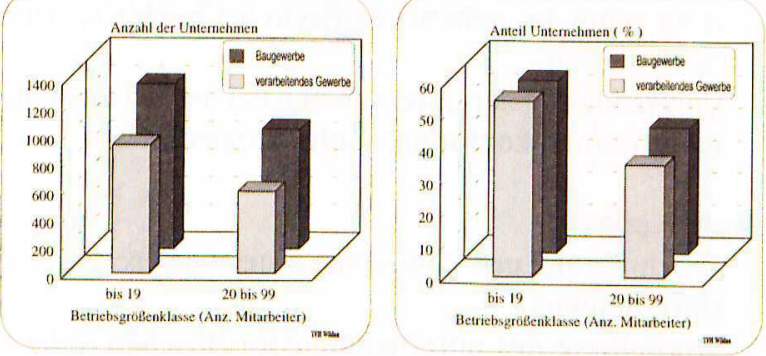

Abb. 6: BetriebsgrößSenverteilung für das verarbeitende Gewerbe und das Baugewerbe im Land Brandenburg 1994 /berechnet nach 1/ (berücksichtigt sind nur Unternehmen bis 99 Mitarbeiter, die relativen Anteile beziehen sich auf die Gesamtzahl der Unternehmen im Land)

Es ist davon auszugehen, daß das Zusammengehen von Unternehmen der gleichen Branche im Unternehmensverbund (Pool) eine besonders effektive Form der Einrichtung von QM-Systemen in KMU darstellt. Erkenntnisse zeigen aber, daß auch der Zusammenschluß von KMU verschiedener aber ähnlich gelagerter Branchen durchaus eine sinnvolle Alternative zur Lösung des oben angedeuteten Problems darstellt (Abb. 7).

\begin{tabular}{|c|c|c|c|c|}
\hline $\begin{array}{l}\text { Ab- } \\
\text { sch. } \\
\text { Nr. }\end{array}$ & Abschnitts-Titel & $\begin{array}{c}\text { Bran } \\
\text { hoch }\end{array}$ & $\begin{array}{l}\text { chensp } \\
\text { mittel }\end{array}$ & $\begin{array}{l}\text { ezifik } \\
\text { klein }\end{array}$ \\
\hline 4 & Verantwortung der Leitung & & & 0 \\
\hline 5 & QM-Elemente & & & 0 \\
\hline 6 & Finanzielle Überleg. zu QMS & & & 0 \\
\hline 7 & Q im Marketing & & 0 & \\
\hline 8 & Q bei Auslegung und Design & & (2) & \\
\hline 9 & Q bei der Beschaffung & & & - \\
\hline 10 & Q von Prozessen & 0 & & \\
\hline 11 & Prozeßlenkung & & ? & \\
\hline 12 & Produktprïfung & & 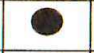 & \\
\hline 13 & Prüfmittelüberwachung & & ( & \\
\hline 14 & Lenkung fehlerhafter Prod. & & & ? \\
\hline 15 & Korrekturmaßnahmen & & & - \\
\hline 16 & Aufgaben nach der Produktion & & ○ & \\
\hline 17 & Qualitätsbezogene Dokumente & & & - \\
\hline 18 & Personal & & & O \\
\hline 19 & Produktsicherheit & & & 0 \\
\hline 20 & Gebrauch statistischer Methoden & & 8 & \\
\hline
\end{tabular}

Abb. 7: Einschätzung der Branchenspezifik (ähnlich gelagerter Branchen) bei Umsetzung von Forderungen des Leitfadens DIN EN ISO 9004-1 in der Muster-QM-Systemdokumentation für KMU der herstellenden Industrie

\section{Projektmanagement im Pool}

Nachfolgend werden Erkenntnisse der Technischen Fachhochschule Wildau in der Projektarbeit im Unternehmensverbund (Poolmodell) bei Einrichtung und Implementierung von QM-Systemen dargestellt.

Die Projektgruppe Qualitätsmanagement der Technischen Fachhochschule (TFH) Wildau hat in den zurïckliegenden Jahren umfangreiche Erkenntnisse und Erfahrungen bei der prozeßnahen Begleitung von KMU zur Einrichtung von normenkonformen QM-Systemen, einschließlich der Vorbereitung der Unternehmen auf eine erfolgreiche Zertifizierung, sammeln können. Die dabei in mehreren Projekten praktizierte Arbeit in Unternehmensverbuinden (Poolmodell) mit zirka $40 \mathrm{KMU}$ erwies sich sowohl inhaltlich/methodisch vorteilhaft (Synergieeffekte) als auch aus wirtschaftlichen Gründen für die beteiligten Unternehmen als günstig. Nachfolgende Darstellungen verdeutlichen Chancen und Grenzen der Zusammenführung von Unternehmen bei der Einrichtung und Implementierung von QM-Systemen.

\section{Chancen:}

- Zugang zum (Querschnitts-)Know-how der Branchen

- Verkuirzung der Zeit für Einrichtung und Implementierung von QM-Systemen,

- Verringerung der finanziellen Aufwendungen je Unternehmen (s. Abb. 8),

- Austausch von Lösungen/Dokumenten nach wechselseitiger Erprobung,

- Bilateraler Wissensaustausch zwischen einzelnen Unternehmen des Pools,

- Strategiebildung des Pools gegenüber Kunden/Lieferanten, 
- günstigere Bedingungen für die Förderung,

- anspornender Wettbewerb im Pool,

- guinstige Voraussetzungen für Zertifizierung.

\section{Grenzen:}

- Abfluß des betrieblichen Know-hows (Prozesse/Abläufe),

- Offenlegung betrieblicher Marktstrategien,

- Verbreitung betriebswirtschaftlicher Kenngrößen,

- Wettbewerbssituation zwischen Unternehmen.

Aufgabe innerhalb des Projektmanagements ist es, die vorteilhaften Bedingungen besonders auszuprägen ohne die bestehenden Grenzen zu iiberschreiten. Dem dient das Arbeitsprinzip der periodischen Beratung im Unternehmensverbund (Pool) und der individuellen Betreuung der beteiligten Unternehmen durch die externe Einrichtung.

Prinzipiell erstreckte/erstreckt sich die Zusammenarbeit im Unternehmensverbund auf die Erarbeitung branchenspezifischer Muster-QM-Dokumentation (QMHandbuch und QM-Verfahrensanweisungen) nach vorangegangener Prozeßkettenanalyse durch die TFH Wildau (Abb. 9, hier als Auszug am Beispiel „Etikettieren der Teile-Nr.").

Die Anpassung dieser QM-Dokumentation für die einzelnen Unternehmen und die Implementierung des QMSystems im Unternehmen wurde/wird durch die Projektgruppe Qualitätsmanagement der TFH Wildau durch prozeßnahe Begleitung (sowohl als Gruppenbetreuung als auch als individuelle Beratung) der an dem Projekt beteiligten Unternehmen unterstuitzt.

Selbstverständlich erfolgte/erfolgt eine fachliche Begleitung der beteiligten Unternehmen bis zur erfolgreichen Zertifizierung ihrer QM-Systeme.

Die prozeßnahe Begleitung der am Projekt beteiligten Unternehmen erfolgt auf der Basis einer detaillierten Aufgabenteilung.

Wichtige Schritte der Einrichtung und Implementierung von QM-Systemen zeigt Abb. 10.

Vor dem eigentlichen Beginn der Zusammenarbeit im Pool sind grundsätzliche Klärungen zu den an dem Projekt teilnehmenden Unternehmen sowie zur Finanzierung des Projektes, einschließlich des Eigenmittelanteils der Unternehmen und eines Förderanteils, vorzunehmen. Schwerpunktaufgaben der Arbeiten in den Phasen des Forschungsprojektes sind:

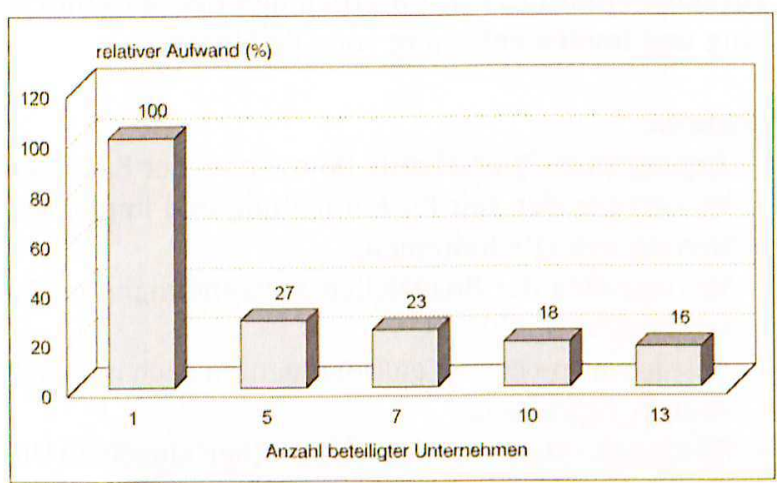

Abb. 8: Relativer finanzieller Aufwand für die externe Begleitung von Unternehmen bei der Einrichtung und Implementierung normenkonformer QMSysteme in Abhängigkeit von der Pool-Größe
Ergebnisse der IST-Zustandsanalyse

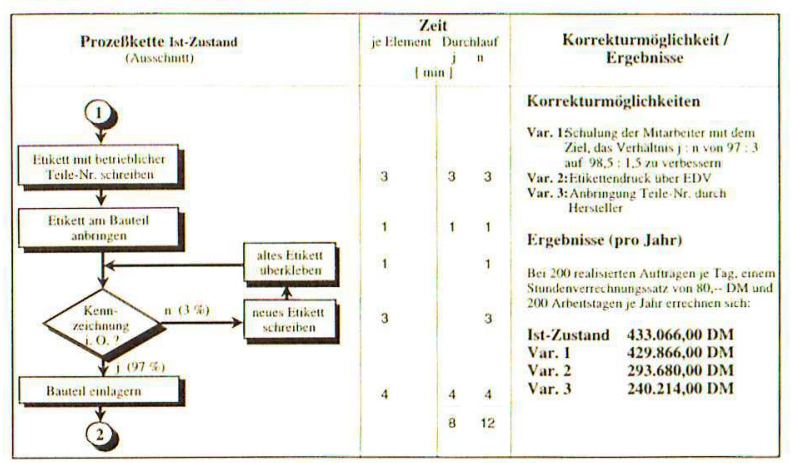

Abb. 9: Auszug aus einer Prozeßkettenanalyse

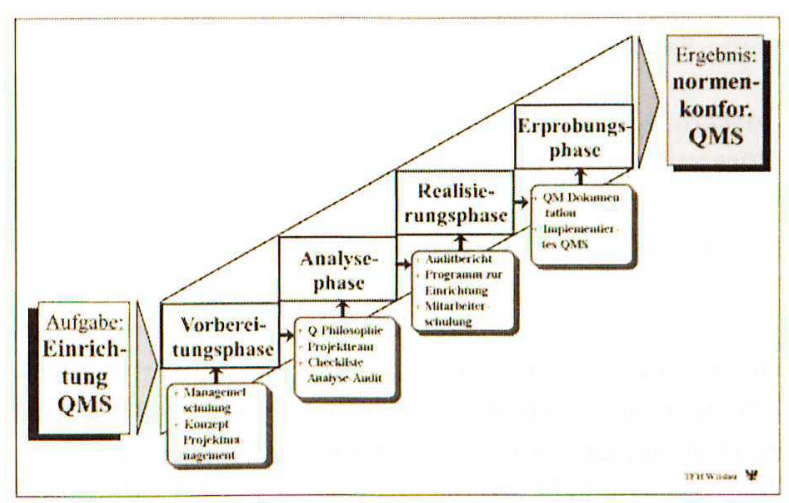

Abb. 10: Phasenmodell zur Einrichtung und Implementierung von QM-Systemen

\section{Vorbereitungsphase}

- Weiterbildung/Schulung der Manager und Qualitätsbeauftragten der beteiligten Unternehmen.

- Entwicklung der Grundsätze der Q-Politik und der QZiele gemeinsam mit den Unternehmen.

- Organisation des Projektmanagements und Befähigung der Unternehmen zur Bildung von Projektteams gemeinsam mit dem externen Berater.

- Vorbereitung der Ist-Zustandsanalyse durch Erarbeitung einer branchenspezifischen Checkliste zum Analyseaudit.

- Unterstiitzung der Unternehmen bei der Entwicklung einer normenkonformen Aufbauorganisation.

\section{Analysephase}

- Durchfuihrung und Bewertung eines Analyseaudits je Unternehmen.

- Erarbeitung des unternehmensspezifischen Ablaufplanes auf der Basis des Auditberichtes zum Analyseaudit. Dieser Ablaufplan detailliert die im Meilensteinplan global beschriebenen Aufgaben für die Realisierungs- und Erprobungsphase.

- Erarbeitung eines Konzeptes für Struktur, Gestaltung und Inhalt der zu erarbeitenden und zu implementierenden QM-Dokumentation.

- Erarbeitung und Bestätigung der Qualitätspolitik und der Qualitätsziele sowie deren Bekanntmachung im Unternehmen.

- Anpassung der Aufbauorganisation an die Forderungen des Normenwerkes des QM und deren Bestätigung.

- Berufung des Beauftragten der obersten Leitung und Einfuihrung in seine Aufgaben sowie Befähigung des Projektteams zur Erfiillung seiner Aufgaben. 


\section{Realisierungsphase}

- Erarbeitung der QM-Musterdokumentation.

- Auf Basis der durch die TFH Wildau bereitgestellten Muster-QMV und des Muster-QMH fuihren die Unternehmen, bei prozeßnaher Begleitung und individueller Betreunng durch die TFH Wildau, die betriebliche Anpassung und Implementierung dieser QMDokumente durch. Parallel dazu erarbeiten sie erforderliche Arbeits- und Prïfanweisungen bzw. passen vorhandene an.

- Realisierung betriebsspezifischer Untersuchungen.

- Durchfuihrung periodischer Anwenderberatungen im Forschungsteam.

\section{Erprobungsphase}

- Bewertung des installierten QM-Systems in den beteiligten Unternehmen durch ein Systemaudit zur Vorbereitung auf die Zertifizierung (Voraudit)

- Ableitung ggf. erforderlicher Korrekturmaßnahmen und deren Begleitung in der Realisierung

- Beratung der Unternehmen zur Zertifizierung

Abb. 11 vermittelt einen qualitativen Überblick zur Entwicklung des arbeitsteiligen Prozesses der Projektarbeit. Die Vorleistungen der TFH Wildau bilden die Basis fuir die Arbeit im Pool. Während die Beratungen im Pool im Laufe der Projektarbeit vom Umfang her zurückgehen, nehmen die Arbeitsanteile im Unternehmen mit fortschreitender Projektlaufzeit zu (eigene Leistungen und individuelle Betreuung durch die TFH Wildau).

\section{Aspekte der Zertifizierung}

Die Projektarbeit im Unternehmensverbund zur Einrichtung und Implementierung von QM-Systemen bietet den beteiligten Unternehmen neben den dargestellten Chancen im eigentlichen Prozeß der Installation von QMSystemen auch direkte Vorteile für die Zertifizierung ihrer QM-Systeme. Als solche werden angesehen:

- einheitliche und gezielte inhaltliche Vorbereitung auf die Zertifizierung,

- kostengünstige Variante, weil:

- von der Struktur und vom Inhalt weitgehende uibereinstimmende QM-Systeme mit „Rationalisierungseffekten " für den Zertifizierer (s. Abb. 12),

- eingeschränkter Leistungsumfang durch Zertifizierungsstelle, z. B. keine Voraudits erforderlich,

- „Mengenrabatt“ durch die Zertifizierungsstelle,

- Vorbereitung weitgehend aus einer Hand,

- rechtzeitige Einbeziehung der Zertifizierungsstelle,

- „Eigendynamik“ im Pool.

Dazu hat es sich als vorteilhaft erwiesen, die Zertifizierungsstelle frühzeitig an die Projektarbeit anzukoppeln. Bei Auswahl der Zertifizierungsstelle selber ist nach Auffassung der Verfassers zu achten auf:

- die Zulassung für die Branche,

- das nationale und internationale Image der Zertifizierungsstelle,

- die Zulassung durch den Gesetzgeber/Kunden,

- die Kosten für die Zertifizierung,

- die „Qualität“ der Dienstleistung Zertifizierung.

\section{Zu erwartende Ergebnisse}

Zielstellung der Zusammenarbeit im Unternehmensverbund ist die Einrichtung und Implementierung normenkonformer QM-Systeme in den an den Projekten beteiligten Unternehmen. Die Projektarbeit schließt die prozeßnahe Begleitung der Unternehmen bis zur Zertifi-

\begin{tabular}{|c|c|c|c|c|}
\hline Vorbereitungsphase & Analysephase & Realisierungsphase & Erprobungsphase & \\
\hline $\begin{array}{l}\text { + Schulung Manager / QB } \\
\text { + Organisation Projekt- } \\
\text { management } \\
\text { + Vorbereitung Analyse- } \\
\text { audit }\end{array}$ & $\begin{array}{l}\text { + Analyseaudit } \\
\text { + Erarb. des unterneh- }\end{array}$ & $\begin{array}{l}\text { + Beratung / Einweisung } \\
\text { QMH / QMV } \\
\text { + Konzept Nachweisdoku- } \\
\text { mentation }\end{array}$ & $\begin{array}{l}+ \text { Vorbereitung der Zerti- } \\
\text { fizierung }\end{array}$ & 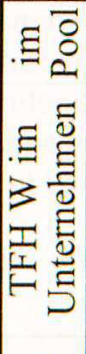 \\
\hline $\begin{array}{l}\text { + Bestätigung Aufbauorg. } \\
\text { + Info. der Mitarbeiter }\end{array}$ & $\begin{array}{l}\text { + Berufung des QB } \\
\text { + Bestätigung / Freigabe der } \\
\text { Q-Politik / Q-Ziele }\end{array}$ & $\begin{array}{l}\text { + Anpassung QMH / QMV } \\
\text { + Erarbeitung AA / PA }\end{array}$ & $\begin{array}{l}\text { + Vorbereitung der Zerti- } \\
\text { fizierung }\end{array}$ & 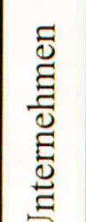 \\
\hline $\begin{array}{l}\text { + Entw. Grundsätze } \\
\text { Q-Politik / Q-Ziele } \\
\text { + Konzept Projekt- } \\
\text { management } \\
\text { + Erarbeitung d. Check- } \\
\text { liste zum Analyseaudit }\end{array}$ & $\begin{array}{l}\text { + Erarb. Rahmen- } \\
\text { arbeitsplan } \\
\text { + Konzept QM-Dokumen- } \\
\text { tation }\end{array}$ & $\begin{array}{l}\text { + Erarb. Muster-QMV } \\
+ \text { Konzept Einbeziehung d. } \\
\text { Zertifizierungsstelle } \\
+ \text { Erarb. Muster-QMH }\end{array}$ & $\begin{array}{l}\text { + Erarb. Checkliste des Vor- } \\
\text { audits } \\
\text { + Auditbericht } \\
\text { + Unterstützung bei Reali- } \\
\text { sierung von eventuellen } \\
\text { Korrekturmaßnahmen }\end{array}$ & 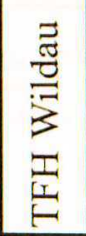 \\
\hline
\end{tabular}

Abb. 11: Ausgewählte Aufgaben im arbeitsteiligen Prozeß der Projektarbeit zur Einrichtung und Implementierung von QM-Systemen 


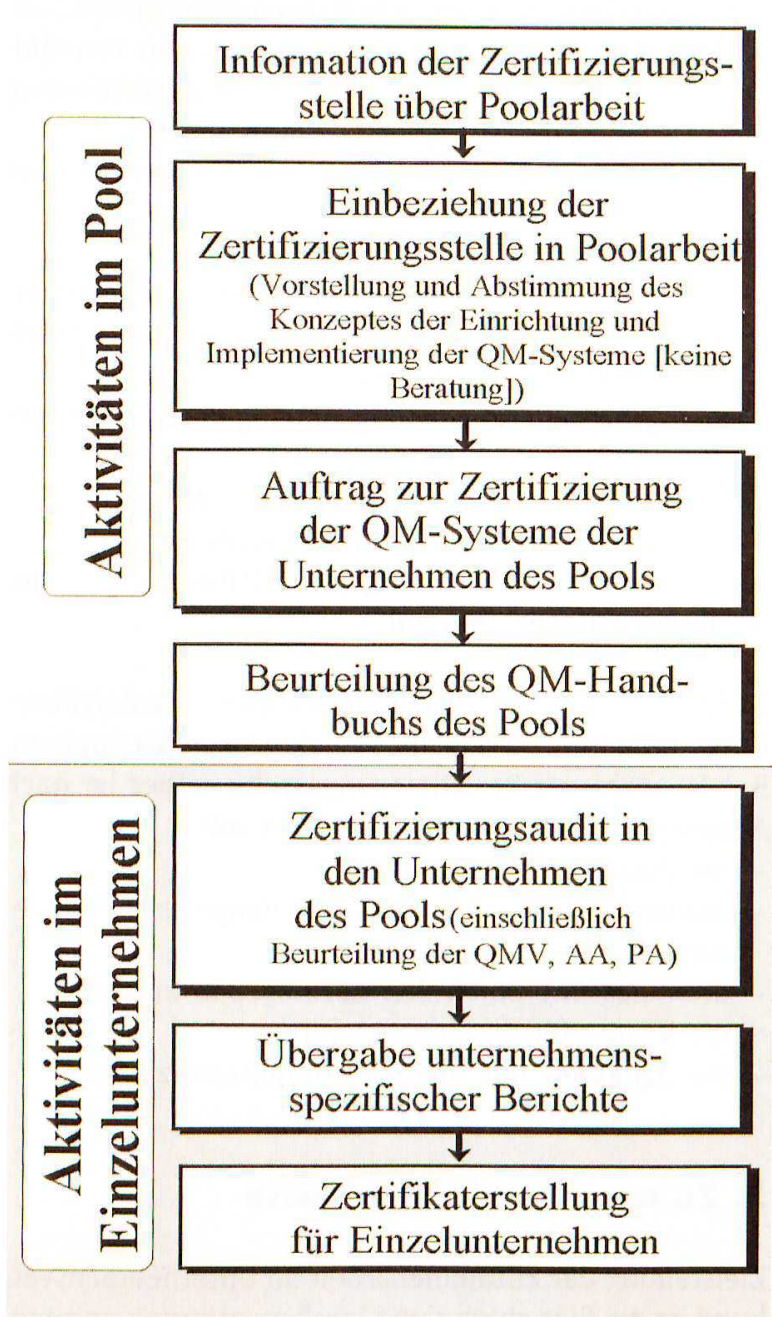

Abb. 12: Aktivitäten der Zertifizierung von QM-Systemen für Poolmodelle

zierung ihrer QM-Systeme ein. Aufgabe ist es, Aspekte des Umweltmanagements direkt in das Qualitätsmanagement zu integrieren.

Nach Auffassung der Verfassers erfordert die derzeitige Situation bei Einrichtung normenkonformer QMSysteme in KMU, gemessen an den nationalen und internationalen Erfordernissen, die Projektarbeit im Unternehmensverbund.

Als entscheidende Vorteile des Zusammenführens von KMU in Projektgruppen werden angesehen:

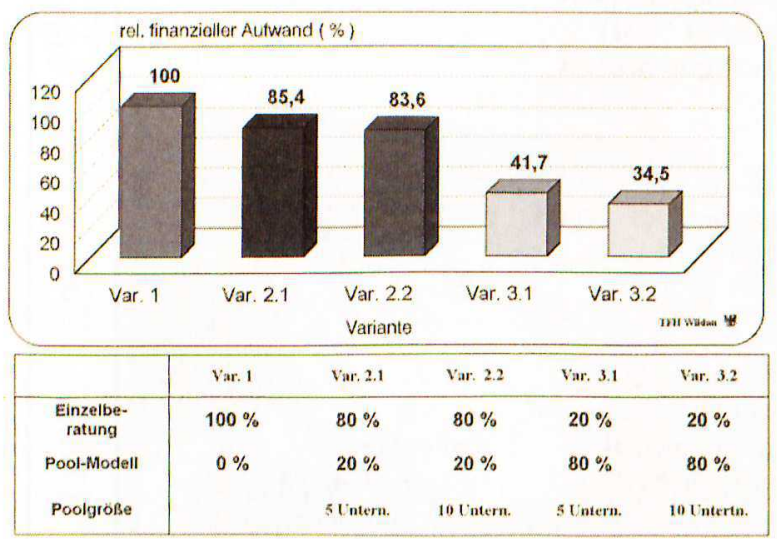

Abb. 13: Relativer finanzieller Aufwand für die externe Unterstützung von KMU bei Einrichtung und Implementierung von QM-Systemen (Berechnungen erfolgten am Beispiel der Angaben in Abb. 6)
- die (schnelle) Überwindung des Rückstandes von KMU bei Gewährleistung und Nachweis der Qualitätsfähigkeit ihrer Unternehmen sowie

- die Senkung des finanziellen Aufwandes für Einrichtung, Implementierung und Zertifizierung von QMSystemen (s. Abb. 13) (u. U. wird dadurch vielen Unternehmen die Realisierung dieser Aufgabe überhaupt erst finanziell möglich).

\section{Literaturverzeichnis}

(1) N. N.: aus Veröffentlichungen des Landesamtes für Datenverarbeitung und Statistik Brandenburg für 1994 (EI1j/94; El4-J/94; EII2-j/94; EIII2-j/94)

(2) N. N.: Management-Wissen Qualität, Wirtschaftswoche Nr. 25 vom 17.6.1994, Seite 91

\section{Verfasser}

Prof. Dr--Ing. habil. Gerd Stegemann

Technische Fachhochschule Wildau Fachbereich Maschinenbau

Tel. (0 33 75) 508-914 